\title{
IMPROVED CONVERGENCE RATES FOR INTERMEDIATE PROBLEMS
}

\author{
CHRISTOPHER BEATTIE AND W. M. GREENLEE
}

\begin{abstract}
Improved convergence rate estimates are derived for a variant of Aronszajn-type intermediate problems that is both computationally feasible and convergent for problems with nontrivial essential spectra. In a previous paper the authors obtained rate of convergence estimates for this method in terms of containment gaps between subspaces. In the present work, techniques for estimating relatively unbounded perturbations are refined in order to apply the Kato-Temple inequalities. This yields convergence rates for the intermediate operator eigenvalues in terms of squares of containment gaps between subspaces. Convergence rate estimates are also obtained for the intermediate problem eigenvectors, and comparisons are made with previously known results for the method of special choice.
\end{abstract}

\section{INTRODUCTION}

The method of intermediate problems of Weinstein and Aronszajn (cf. [33]) provides a systematic method for generating improvable bounds for eigenvalues of selfadjoint operators which are complementary to the Rayleigh-Ritz bounds. Convergence criteria for Weinstein's method date back to Aronszajn and Weinstein $[2,3]$ for problems with compact resolvent, and for Aronszajn's method to Aronszajn [1] and Bazley and Fox [5] for problems with compact resolvent and relatively bounded perturbations. The approximation method of Weinberger $[30,32]$, which is closely related to the method of intermediate problems (cf. [16]), is the first method for which convergence criteria were given in settings admitting a nontrivial essential spectrum. For intermediate problem applications that admit nontrivial essential spectra and perturbations that are not relatively bounded-as occur, e.g., in many quantum mechanical eigenvalue problemsconvergence results are relatively recent, including those of Beattie [9], Beattie and Greenlee [10], Brown [13, 14], and Greenlee [19].

Convergence rates for eigenvalues of intermediate problems were first derived by Weinberger [29] for Weinstein's method. Subsequently, Fix [15] and Birkhoff

Received by the editor October 25, 1989.

1991 Mathematics Subject Classification. Primary 49R15, 65N15, 65N25; Secondary 35P15.

The work of the first author was partially supported by AFOSR Grant 84-0326 while he was a visiting fellow at the Minnesota Supercomputer Institute and Department of Computer Science, University of Minnesota.

The work of the second author was partially supported by NSF Grant INT-8413270 during a visit to the Mathematisch Instituut, Rijksuniversiteit Utrecht, and by the Institute for Mathematics and its Applications, University of Minnesota, with funds provided by the National Science Foundation. 
and Fix [12] obtained convergence rates for Bazley's method of special choice [4] in the case of bounded perturbations, and Poznyak [25, 26] derived rate of convergence results for variants of Aronszajn's method with relatively bounded perturbations of the base operator. These results on convergence rates all depend on compactness in some fashion. Even less is known about convergence rates for eigenprojections and eigenvectors. Estimates such as those found in Kato [22], Weinberger [31], and Bazley and Fox [6], depend on both upper and lower bound information, while in $[12,15]$ convergence rates for the method of special choice have been obtained in the case of bounded perturbations.

Herein we refine the rate of convergence estimates of our recent paper [11] for a particular method of Aronszajn type known variously as "truncation including the remainder" $[9,10]$, or as "Aronszajn's method with a truncated base problem" [19]. This method was first discussed by Bazley and Fox [8] who verified two interesting features of the method. First, with an appropriate selection of trial vectors, this method dominates Weinberger's method and, secondly, this method is always dominated by Aronszajn's method without truncation. Thus, we simultaneously obtain convergence rates for other variants of Aronszajn's method as well. Truncation including the remainder is the only method of Aronszajn type known to be both computationally feasible and convergent for problems with nontrivial essential spectra. This method also requires relatively unbounded perturbations of the base operator whenever the operator of interest is itself unbounded, and, like Weinberger's method, can be employed for constraint problems (cf. [19]).

In $\S 2$ we formulate Aronszajn's method with a truncated base operator, and review previous results on convergence that are relevant to the sequel. The theorems of $\S 3$ depend on an estimate of Kato [22]. Theorem 3.1 effectively squares the rate of convergence estimate for eigenvalues obtained in [11]. Both of these estimates depend strong!y on implementation of a construction of [19] to estimate relatively unbounded perturbations by means of abstractly defined bounded perturbations. While this device enables one to estimate relatively unbounded perturbations, it does not immediately yield convergence information for the eigenvectors actually constructed in the approximation method, contrary to a remark in [19]. Then, in Theorem 3.2, we derive estimates for eigenprojections and eigenvectors. While these estimates may be somewhat crude, they appear to be the first that are applicable in the presence of nontrivial essential spectra and relatively unbounded perturbations without prior upper bound information. Finally, in $\S 4$ we extend the technique of [11] for implementing the convergence rate estimates in differential eigenvalue problems so as to apply Theorems 3.1 and 3.2. For illustration we directly compare rates of convergence obtained from our theorems with rates derived in [12, 15] for approximation of Sturm-Liouville problems by the method of special choice, and provide two computational examples that illustrate the derived rates.

\section{THE APPROXIMATION METHOD}

Let $\mathfrak{H}$ be a separable complex Hilbert space with norm $\|u\|$ and inner product $\langle u, v\rangle$. Let $A$ be a selfadjoint operator with domain $\mathscr{D}(A)$ dense in $\mathfrak{H}$. We suppose that $A$ is bounded below with spectrum that begins with isolated eigenvalues of finite multiplicity, 


$$
\lambda_{1}(A) \leq \lambda_{2}(A) \leq \cdots \leq \lambda_{\infty}(A)
$$

and corresponding orthonormal eigenvectors $u_{1}, u_{2}, \ldots$ Here, $\lambda_{\infty}(A)$ denotes the least point of the essential spectrum of $A$, where by convention we set $\lambda_{\infty}(A)=\infty$ if the essential spectrum of $A$ is empty. The closure of the quadratic form $\langle A u, u\rangle$ is denoted by $a(u)$.

To apply the method of intermediate problems, we require knowledge of another closed quadratic form $a_{0}(u)$, satisfying $a_{0}(u) \leq a(u)$ for all $u \in \mathscr{D}(a)$. Furthermore, we require that the spectral problem for the selfadjoint operator $A_{0}$ corresponding to $a_{0}$ is solved explicitly, and that the spectrum of $A_{0}$ also begins with isolated eigenvalues of finite multiplicity,

$$
\lambda_{1}\left(A_{0}\right) \leq \lambda_{2}\left(A_{0}\right) \leq \cdots \leq \lambda_{\infty}\left(A_{0}\right),
$$

with corresponding orthonormal eigenvectors $u_{1}^{0}, u_{2}^{0}, \ldots$. The second monotonicity principle implies that $\lambda_{\infty}\left(A_{0}\right) \leq \lambda_{\infty}(A)$, and that for each $i$ such that $\lambda_{i}(A)<\lambda_{\infty}\left(A_{0}\right), \lambda_{i}\left(A_{0}\right)$ exists and $\lambda_{i}\left(A_{0}\right) \leq \lambda_{i}(A)$. Without loss of generality we may assume that the difference between $a$ and $a_{0}$ is strictly positive, i.e.,

$$
\hat{a}(u)=a(u)-a_{0}(u) \geq \alpha\|u\|^{2},
$$

for some $\alpha>0$ and all $u \in \mathscr{D}(a)$.

Now pick a real number $\gamma$ satisfying $\lambda_{1}\left(A_{0}\right)<\gamma \leq \lambda_{\infty}\left(A_{0}\right)$, with the restriction that $\gamma<\lambda_{\infty}\left(A_{0}\right)$ if $A_{0}$ has an infinity of eigenvalues below $\lambda_{\infty}\left(A_{0}\right)$. Define the truncation of $A_{0}$ at $\gamma$ by

$$
A_{0}^{(\gamma)}=A_{0} E_{\gamma-}\left[A_{0}\right]+\gamma\left(I-E_{\gamma-}\left[A_{0}\right]\right),
$$

where $E_{\lambda}\left[A_{0}\right]$ is the right-continuous resolution of the identity for $A_{0}$. Observe that $A_{0}^{(\gamma)}$ has the same action as $A_{0}$ on the finite-dimensional subspace

$$
\mathscr{U}_{0}^{\gamma}=\mathscr{R}\left(E_{\gamma^{-}}\left[A_{0}\right]\right)=E_{\gamma^{-}}\left[A_{0}\right] \cdot \mathfrak{H},
$$

and acts as scalar multiplication by $\gamma$ on vectors in $\left(\mathscr{U}_{0}^{\gamma}\right)^{\perp}$. The corresponding quadratic form $a_{0}^{(\gamma)}$ may be used to define a second positive form,

$$
\tilde{a}(u)=a(u)-a_{0}^{(\gamma)}(u) \geq \hat{a}(u) \geq \alpha\|u\|^{2} .
$$

Obviously, $\mathscr{D}(\tilde{a})=\mathscr{D}(a), \tilde{a}$ is a closed quadratic form, and the corresponding selfadjoint operator is given by $\tilde{A}=A-A_{0}^{(\gamma)}$ on $\mathscr{D}(\tilde{A})=\mathscr{D}(A)$.

The method of approximation to be studied is simply Aronszajn's method with the truncated base operator $A_{0}^{(\gamma)}$ instead of the original base operator $A_{0}$ (cf. $[18,19]$ ). The method proceeds by selecting a set of trial vectors $\left\{p_{i}\right\}_{i=1}^{\infty} \subset$ $\mathscr{D}(\widetilde{A})$ and defining for each $n$

$$
P_{n} u=\sum_{i, j=1}^{n}\left\langle u, \tilde{A} p_{i}\right\rangle b_{i j} p_{j}
$$

where $\left[b_{i j}\right]$ is the matrix inverse to $\left[\left\langle p_{i}, \tilde{A} p_{j}\right\rangle\right]_{i, j=1}^{n}$. Then $P_{n}$ is the projection onto $\mathscr{P}_{n}=\operatorname{span}\left\{p_{1}, \ldots, p_{n}\right\}$ that is orthogonal with respect to the inner product induced by $\tilde{a}$. 
For each $n$, define the intermediate form

$$
a_{n}(u)=a_{0}^{(\gamma)}(u)+\tilde{a}\left(P_{n} u\right)
$$

for $u \in \mathscr{D}\left(a_{n}\right)=\mathfrak{H}$, with the corresponding selfadjoint operator

$$
A_{n}=A_{0}^{(\gamma)}+\widetilde{A} P_{n}
$$

By construction,

$$
a_{0}^{(\gamma)}(u) \leq a_{n}(u) \leq a_{n+1}(u) \leq a(u)
$$

for all $u \in \mathscr{D}(a)$, and thus the second monotonicity principle provides

$$
\lambda_{i}\left(A_{0}\right)=\lambda_{i}\left(A_{0}^{(\gamma)}\right) \leq \cdots \leq \lambda_{i}\left(A_{n}\right) \leq \lambda_{i}\left(A_{n+1}\right) \leq \cdots \leq \lambda_{i}(A),
$$

for all $i$ such that $\lambda_{i}(A)<\gamma$. Thus, the intermediate operators, $\left\{A_{n}\right\}$, have eigenvalues that provide improving lower bounds to the eigenvalues of $A$ as the index $n$ is increased. For discussion of practical issues involving computation of intermediate operator eigenvalues, see [7,32,33]. Convergence criteria for this approximation method are given by the following theorem [10].

Theorem 2.1. If the set of vectors $\left\{p_{i}\right\}_{i=1}^{\infty}$ is complete in $\mathscr{D}(\widetilde{A})$ with respect to the norm $\|\tilde{A} u\|$, then $\lim _{n \rightarrow \infty} \lambda_{i}\left(A_{n}\right)=\lambda_{i}(A)$ for each $i$ such that $\lambda_{i}(A)<\gamma$, $\lim _{n \rightarrow \infty} \lambda_{i}\left(A_{n}\right) \geq \gamma$ for each $i$ such that $\lambda_{i}(A) \geq \gamma$, and $\left\|E_{\lambda}[A]-E_{\lambda}\left[A_{n}\right]\right\| \rightarrow 0$ as $n \rightarrow \infty$ for every $\lambda<\gamma$.

Our convergence rate estimates are motivated by the following conditions for exactness (cf. [11]). Define $\mathscr{U}^{\gamma}=\mathscr{R}\left(E_{\gamma^{-}}[A]\right)=E_{\gamma^{-}}[A] \cdot \mathfrak{H}$, and let $P_{n}^{*}$ denote the adjoint of $P_{n}$ in $\mathfrak{H}$, as usual.

Theorem 2.2. Suppose the convergence criteria of Theorem 2.1 are satisfied. If for all $n \geq N$,

$$
\mathscr{R}\left(P_{n}^{*}\right) \supset \mathscr{U}^{\gamma}+\mathscr{U}_{0}^{\gamma},
$$

then for all $n \geq N$,

$$
\mathscr{R}\left(P_{n}\right) \supset \mathscr{U}^{\gamma},
$$

and furthermore, when (2.3) holds for $n \geq N$, then also $\lambda_{i}\left(A_{n}\right)=\lambda_{i}(A)$ for all $n \geq N$ and each $i$ such that $\lambda_{i}(A)<\gamma$.

It is thus appropriate to seek rate of convergence estimates in terms of a notion of separation between the "approximating subspaces", $\mathscr{R}\left(P_{n}^{*}\right)$, and the "exact subspaces" $\mathscr{U}^{\gamma}+\mathscr{U}_{0}^{\gamma}$. An appropriate measure of separation is the containment gap for a subspace $\mathscr{N}$ by a subspace $\mathscr{M}$.

Definition 2.3. Let $\mathscr{M}$ and $\mathscr{N}$ be closed subspaces of $\mathfrak{H}$ with $\operatorname{dim} \mathscr{N}>0$. The containment gap for $\mathscr{N}$ by $\mathscr{M}$ is

$$
\delta_{\mathscr{N}}(\mathscr{M})=\sup _{0 \neq u \in \mathscr{N}} \frac{\|(I-Q) u\|}{\|u\|}=\|(I-Q) P\|,
$$

where $Q$ is the orthogonal projection onto $\mathscr{M}$ and $P$ is the orthogonal projection onto $\mathcal{N}$.

Unlike the gap of [23, p. 197], $\delta_{\mathscr{N}}(\mathscr{M})$ is not symmetric in $\mathscr{M}$ and $\mathscr{N}$, and $\delta_{\mathscr{N}}(\mathscr{M})=0$ if and only if $\mathscr{M} \supset \mathscr{N}$. We now state the basic estimate of [11]. Herein we let $\mathscr{P}_{n}=\mathscr{R}\left(P_{n}\right)$, and then one has $\widetilde{A} \mathscr{P}_{n}=\mathscr{R}\left(P_{n}^{*}\right)$. 
Theorem 2.4. There exists a constant $K>0$, independent of $n$, such that for each $i$ satisfying $\lambda_{i}(A)<\gamma$,

$$
\left|\lambda_{i}(A)-\lambda_{i}\left(A_{n}\right)\right| \leq K\left\{\delta_{\mathscr{L}}\left(\tilde{A}_{\mathscr{P}_{n}}\right)+\delta_{\mathscr{M}}\left(\tilde{A} \mathscr{P}_{n}\right)\right\}
$$

where $\mathscr{L}=\mathscr{U}_{0}^{\gamma}$ and $\mathscr{M}=\mathscr{U}^{\gamma}$.

Observe that the convergence criteria of Theorem 2.1 are not hypothesized in Theorem 2.4. Thus Theorem 2.4 immediately yields the following theorem, which is reminiscent of conditions for convergence of Weinberger's method [ 30 , 32]. Herein, $\operatorname{cl}(Z)$ represents the closure of a set $Z$ in the underlying Hilbert space $\mathfrak{H}$.

Theorem 2.5. If $\mathfrak{c l}\left(\bigcup_{n=1}^{\infty} \tilde{A} \mathscr{P}_{n}\right) \supset \mathscr{U}^{\gamma}+\mathscr{U}_{0}^{\gamma}$, then $\lim _{n \rightarrow \infty} \lambda_{i}\left(A_{n}\right)=\lambda_{i}(A)$ for all $i$ such that $\lambda_{i}(A)<\gamma$.

To improve on the estimate of Theorem 2.4, we use the following eigenvalue estimate due to Kato [22]. The estimate (2.5) for eigenprojections is contained within Kato's proof of Lemma 18.4 in [22], and is also developed in [18] together with the explicit eigenvector bound (2.6). As usual, $\delta_{k l}$ denotes the Kronecker symbol.

Theorem 2.6. For $S$ a selfadjoint operator in $\mathfrak{H}$, let $v_{1}, \ldots, v_{m} \in \mathscr{D}(S)$ be $m$ vectors for which

$$
\begin{gathered}
\left\langle v_{k}, v_{l}\right\rangle=\delta_{k l}, \quad\left\langle S v_{k}, v_{l}\right\rangle=\eta_{k} \delta_{k l}, \quad k, l=1, \ldots, m, \\
\eta_{1} \leq \eta_{2} \leq \cdots \leq \eta_{m},
\end{gathered}
$$

and let $\theta_{k}=\left\|\left(S-\eta_{k}\right) v_{k}\right\|, k=1, \ldots, m$. Further, let $(c, d)$ be an interval whose intersection with the spectrum of $S$ consists solely of eigenvalues of total multiplicity at most $m$. If $c<\eta_{1}, \eta_{m}<d$, and

$$
\sum_{k=1}^{m} \frac{\theta_{k}^{2}}{\left(\eta_{k}-c\right)\left(d-\eta_{k}\right)}<1
$$

then there are exactly $m$ eigenvalues $\Lambda_{1} \leq \Lambda_{2} \leq \cdots \leq \Lambda_{m}$ of $S$ in the interval $(c, d)$, and they satisfy

$$
-\sum_{k=l}^{m} \frac{\theta_{k}^{2}}{d-\eta_{k}} \leq \Lambda_{l}-\eta_{l} \leq \sum_{k=1}^{l} \frac{\theta_{k}^{2}}{\eta_{k}-c}, \quad l=1, \ldots, m .
$$

Furthermore, if $P$ is the orthogonal projection onto the m-dimensional subspace spanned by the eigenvectors of $S$ corresponding to $\Lambda_{1}, \ldots, \Lambda_{m}$, and $\delta_{k}=\min \left\{\eta_{k}-c, d-\eta_{k}\right\}$, then

$$
\left\|P v_{k}-v_{k}\right\| \leq \theta_{k} / \delta_{k}, \quad k=1, \ldots, m .
$$

If $m=1$ and $w_{1}$ is the corresponding eigenvector of $S$ normalized by $\left\|w_{1}\right\|=1$ and $\left\langle w_{1}, v_{1}\right\rangle \geq 0$, then

$$
\left\|w_{1}-v_{1}\right\|^{2} \leq 2 \theta_{1}^{2} / \delta_{1}^{2}\left[1+\left(1-\left(\theta_{1}^{2} / \delta_{1}^{2}\right)\right)^{1 / 2}\right] .
$$

\section{Convergence Rates}

We begin with a rate of convergence estimate for the eigenvalues of $A$ below $\gamma$. The proof is based on an implementation of Theorem 2.6 by means of a 
construction of $[11,19]$, which is also basic to the proof of Theorem 2.4. We sketch this construction in the course of the proof, but full details are available in $[11,19]$.

Theorem 3.1. Let $\lambda_{h}(A)=\lambda_{h+1}(A)=\cdots=\lambda_{h+m-1}(A)$ be an eigenvalue of $A$ below $\gamma$ with multiplicity $m$ and corresponding eigenspace $\mathscr{S}$. Further, assume that the convergence criteria of either Theorem 2.1 or Theorem 2.5 are satisfied. Then for each $j=h, h+1, \ldots, h+m-1$, and all $n$ large enough,

$$
\left|\lambda_{j}(A)-\lambda_{j}\left(A_{n}\right)\right| \leq K\left\{\delta_{\mathscr{M}}^{2}\left(\tilde{A}_{n}\right)+\delta_{\mathscr{N}}^{2}\left(\tilde{A}_{n}\right)\right\},
$$

where $\mathscr{M}=\mathscr{U}^{\gamma}, \mathscr{N}=\mathscr{S}+\mathscr{U}_{0}^{\gamma}$, and $K$ is independent of $n$.

Proof. Consider the truncation of $A$ at $\gamma$,

$$
A^{(\gamma)}=A E_{\gamma^{-}}[A]+\gamma\left(I-E_{\gamma^{-}}[A]\right),
$$

and introduce the auxiliary selfadjoint operator,

$$
A_{n}^{\prime}=A_{0}^{(\gamma)}+P_{n}^{*}\left(A^{(\gamma)}-A_{0}^{(\gamma)}\right) P_{n}
$$

It follows easily that $\left\langle A_{n}^{\prime} u, u\right\rangle \leq\left\langle A_{n} u, u\right\rangle$ for all $u \in \mathfrak{H}$, so

$$
\left|\lambda_{j}(A)-\lambda_{j}\left(A_{n}\right)\right| \leq\left|\lambda_{j}(A)-\lambda_{j}\left(A_{n}^{\prime}\right)\right| \leq\left|\lambda_{j}(A)-\eta_{k}\right|+\left|\eta_{k}-\lambda_{j}\left(A_{n}^{\prime}\right)\right|,
$$

where $\eta_{k}$ will be specified below. Evidently, it will suffice to prove that the last two terms are dominated by the right-hand side of (3.1).

We now assume that $A$ is bounded, and will treat the unbounded case later. We will employ Theorem 2.6 with $S=A_{n}^{\prime},(c, d)$ any isolating interval for $\lambda_{h}(A)$, and $\left\{v_{k}\right\}_{k=1}^{m}$ an orthonormal basis for the eigenspace $\mathscr{S}$ which also diagonalizes the $m \times m$ matrix $\left[\left\langle A_{n}^{\prime} v_{k}, v_{l}\right\rangle\right]$, yielding matrix eigenvalues $\eta_{1} \leq \eta_{2} \leq \cdots \leq \eta_{m}$ given by $\eta_{k}=\left\langle A_{n}^{\prime} v_{k}, v_{k}\right\rangle, k=1, \ldots, m$. Then $\theta_{k}=$ $\left\|\left(A_{n}^{\prime}-\eta_{k}\right) v_{k}\right\|$, for $k=1, \ldots, m$. The vectors $v_{k}$ and the numbers $\eta_{k}, \theta_{k}$ may depend on $n$, but we are free to use a notation that suppresses that dependence.

Thus,

$$
\begin{aligned}
\eta_{k}-\lambda_{h}(A)= & \eta_{k}-\left\langle A^{(\gamma)} v_{k}, v_{k}\right\rangle=\left\langle\left(A_{n}^{\prime}-A^{(\gamma)}\right) v_{k}, v_{k}\right\rangle \\
= & \left\langle\left(A_{0}^{(\gamma)}+P_{n}^{*}\left(A^{(\gamma)}-A_{0}^{(\gamma)}\right) P_{n}-A^{(\gamma)}\right) v_{k}, v_{k}\right\rangle \\
= & \left\langle\left[\left(P_{n}^{*}-I\right)\left(A^{(\gamma)}-A_{0}^{(\gamma)}\right)\left(P_{n}-I\right)+\left(A^{(\gamma)}-A_{0}^{(\gamma)}\right)\left(P_{n}-I\right)\right.\right. \\
& \left.\left.+\left(P_{n}^{*}-I\right)\left(A^{(\gamma)}-A_{0}^{(\gamma)}\right)\right] v_{k}, v_{k}\right\rangle .
\end{aligned}
$$

Now,

$$
\begin{aligned}
\left\langle\left( P_{n}^{*}\right.\right. & \left.-I)\left(A^{(\gamma)}-A_{0}^{(\gamma)}\right) v_{k}, v_{k}\right\rangle \\
& =\left\langle\left(P_{n}^{*}-I\right)\left(I-Q_{n}\right)\left(A^{(\gamma)}-A_{0}^{(\gamma)}\right) v_{k}, v_{k}\right\rangle \\
& =\left\langle\left(I-Q_{n}\right)\left(A^{(\gamma)}-A_{0}^{(\gamma)}\right) v_{k},\left(P_{n}-I\right) v_{k}\right\rangle,
\end{aligned}
$$

where $Q_{n}$ is the orthogonal projection onto $\mathscr{R}\left(P_{n}^{*}\right)=\widetilde{A} \mathscr{P}_{n}$. Hence,

$$
\begin{aligned}
& \left|\left\langle\left(P_{n}^{*}-I\right)\left(A^{(\gamma)}-A_{0}^{(\gamma)}\right) v_{k}, v_{k}\right\rangle\right| \\
& \quad \leq\left\|\left(I-Q_{n}\right)\left(A^{(\gamma)}-A_{0}^{(\gamma)}\right) v_{k}\right\| \cdot\left\|\left(I-P_{n}\right) v_{k}\right\| \\
& \quad \leq\left(\gamma-\lambda_{1}\left(A_{0}\right)\right) \delta_{\mathcal{N}}\left(\tilde{A} \mathscr{P}_{n}\right)\left\|\left(I-P_{n}\right) v_{k}\right\|,
\end{aligned}
$$


where $\mathscr{N}=\mathscr{S}+\mathscr{U}_{0}^{(\gamma)}$. The last inequality uses the facts that $\left\|v_{k}\right\|=1$, $\left(A^{(\gamma)}-A_{0}^{(\gamma)}\right) v_{k}=\left(\lambda_{h}(A)-A_{0}^{(\gamma)}\right) v_{k} \in \mathscr{N}$, and $\left\|A^{(\gamma)}-A_{0}^{(\gamma)}\right\| \leq \gamma-\lambda_{1}\left(A_{0}\right)$. Now observe that, since $\widetilde{A}\left(I-P_{n}\right)$ is selfadjoint,

$$
\left\|\left(I-P_{n}\right) v_{k}\right\| \leq \alpha^{-1}\left\|\widetilde{A}\left(I-P_{n}\right) v_{k}\right\|=\alpha^{-1}\left\|\left(I-P_{n}^{*}\right) \tilde{A} v_{k}\right\| .
$$

Since $P_{n}$ and $I-P_{n}$ are $\tilde{a}$-symmetric for each $n$, and $\tilde{a}$ is bounded, we have the uniform bound $\left\|P_{n}-I\right\|=\left\|P_{n}^{*}-I\right\| \leq \kappa$, where $\kappa=\left\|\widetilde{A}^{1 / 2}\right\| \cdot\left\|\widetilde{A}^{-1 / 2}\right\|$ with $\widetilde{A}^{1 / 2}$ the unique positive definite square root of $\tilde{A}$. Hence,

$$
\begin{aligned}
\left\|\left(I-P_{n}\right) v_{k}\right\| & \leq \alpha^{-1}\left\|\left(I-P_{n}^{*}\right)\left(I-Q_{n}\right) \tilde{A} v_{k}\right\| \\
& \leq \alpha^{-1} \kappa\left\|\left(I-Q_{n}\right) \tilde{A} v_{k}\right\| \leq \alpha^{-1} \kappa\left(\gamma-\lambda_{1}\left(A_{0}\right)\right) \delta_{\mathcal{N}}\left(\tilde{A} \mathscr{P}_{n}\right),
\end{aligned}
$$

since $\left\|v_{k}\right\|=1$ and $\tilde{A} v_{k}=\left(A-A_{0}^{(\gamma)}\right) v_{k}=\left(\lambda_{h}(A)-A_{0}^{(\gamma)}\right) v_{k}$. Thus,

$$
\begin{aligned}
\left|\left\langle\left(P_{n}^{*}-I\right)\left(A^{(\gamma)}-A_{0}^{(\gamma)}\right) v_{k}, v_{k}\right\rangle\right| & =\left|\left\langle\left(A^{(\gamma)}-A_{0}^{(\gamma)}\right)\left(P_{n}-I\right) v_{k}, v_{k}\right\rangle\right| \\
& \leq \alpha^{-1} \kappa\left(\gamma-\lambda_{1}\left(A_{0}\right)\right)^{2} \delta_{\mathscr{N}}^{2}\left(\widetilde{A} \mathscr{P}_{n}\right),
\end{aligned}
$$

and similarly,

$$
\begin{aligned}
\left|\left\langle\left(P_{n}^{*}-I\right)\left(A^{(\gamma)}-A_{0}^{(\gamma)}\right)\left(P_{n}-I\right) v_{k}, v_{k}\right\rangle\right| \\
\quad=\left|\left\langle\left(A^{(\gamma)}-A_{0}^{(\gamma)}\right)\left(P_{n}-I\right) v_{k},\left(P_{n}-I\right) v_{k}\right\rangle\right| \\
\quad \leq\left(\gamma-\lambda_{1}\left(A_{0}\right)\right)\left\|\left(P_{n}-I\right) v_{k}\right\|^{2} \leq \alpha^{-2} \kappa^{2}\left(\gamma-\lambda_{1}\left(A_{0}\right)\right)^{3} \delta_{\mathcal{N}}^{2}\left(\tilde{A} \mathscr{P}_{n}\right) .
\end{aligned}
$$

Thus, $\left|\eta_{k}-\lambda_{h}(A)\right| \leq K_{1} \delta_{\mathcal{N}}^{2}\left(\tilde{A} P_{n}\right)$, for some constant $K_{1}$ independent of $n$.

Note that

$$
\begin{aligned}
\theta_{k} & =\left\|\left[A^{(\gamma)}+\left(A_{n}^{\prime}-A^{(\gamma)}\right)-\left(\lambda_{h}(A)+\left\langle\left(A_{n}^{\prime}-A^{(\gamma)}\right) v_{k}, v_{k}\right\rangle\right)\right] v_{k}\right\| \\
& =\left\|\left[A_{n}^{\prime}-A^{(\gamma)}-\left\langle\left(A_{n}^{\prime}-A^{(\gamma)}\right) v_{k}, v_{k}\right\rangle\right] v_{k}\right\| \\
& \leq\left\|A_{n}^{\prime}-A^{(\gamma)}\right\| .
\end{aligned}
$$

Furthermore, Lemmas 3.1 and 3.2 of [11] together yield

$$
\left\|A_{n}^{\prime}-A^{(\gamma)}\right\| \leq C\left\{\delta_{\mathscr{L}}\left(\widetilde{A} \mathscr{P}_{n}\right)+\delta_{\mathscr{M}}\left(\widetilde{A} \mathscr{P}_{n}\right)\right\},
$$

where $\mathscr{L}=\mathscr{U}_{0}^{\gamma}, \mathscr{M}=\mathscr{U}^{\gamma}$, and the positive constant $C$ is independent of $n$, and so

$$
\theta_{k}^{2} \leq 2 C^{2}\left\{\delta_{\mathscr{L}}^{2}\left(\tilde{A}_{n}\right)+\delta_{\mathscr{M}}^{2}\left(\tilde{A}_{n}\right)\right\}
$$

As $n \rightarrow \infty$, the convergence criteria imply that $\theta_{k} \rightarrow 0$ and $\eta_{k} \rightarrow \lambda_{h}(A)$ for each $k=1,2, \ldots, m$ and that $\lambda_{j}\left(A_{n}^{\prime}\right) \rightarrow \lambda_{h}(A)$ for each $j=h, h+1, \ldots$, $h+m-1$. The hypotheses of Theorem 2.6 hold for sufficiently large $n$, hence for each $j=h, h+1, \ldots h+m-1$ there is an index $1 \leq k(j) \leq m$ and a constant $K_{2}$ independent of $n$ such that

$$
\left|\eta_{k(j)}-\lambda_{j}\left(A_{n}^{\prime}\right)\right| \leq K_{2} \theta_{k(j)}^{2} \leq 2 K_{2} C\left\{\delta_{\mathscr{L}}^{2}\left(\tilde{A} P_{n}\right)+\delta_{\mathscr{M}}^{2}\left(\tilde{A} P_{n}\right)\right\} .
$$

The conclusion (3.1) now follows immediately.

We now admit the possibility that $A$ is unbounded and observe that the above proof does not apply, since in that case there may not be a bound independent of $n$ for $\left\|P_{n}-I\right\|=\left\|P_{n}^{*}-I\right\|$. This difficulty will be circumvented by employing a device of [19] which was also used in [11]. We assume without loss of generality 
that the quadratic form $\left\langle A_{0}^{(\gamma)} u, u\right\rangle$ is nonnegative and consider the auxiliary bounded selfadjoint operator

$$
\widetilde{\widetilde{A}}=A^{(\mu)}-A_{0}^{(\gamma)},
$$

where $\mu$ is some fixed positive number satisfying $\mu \geq \gamma(1+(2 \gamma / \alpha))+(\alpha / 2)$. Then the corresponding quadratic form satisfies $\tilde{\tilde{a}}(u) \geq(\alpha / 2)\|u\|^{2}$ for all $u \in \mathfrak{H}$ (cf. [19]). It follows that

$$
a^{(\mu)}(u)=a_{0}^{(\gamma)}(u)+\tilde{\tilde{a}}(u),
$$

and we apply Aronszajn's method to this decomposition of $a^{(\mu)}$ in the following way.

Given the trial vectors $\left\{p_{i}\right\}_{i=1}^{\infty} \subset \mathscr{D}(A)$, generate $\left\{\hat{p}_{i}\right\}_{i=1}^{\infty}$ by $\hat{p}_{i}=\tilde{\widetilde{A}}^{-1} \tilde{A} p_{i}$ for each $i=1,2, \ldots$. Define projections $\widehat{P}_{n}$ onto $\widehat{\mathscr{P}}_{n}=\operatorname{span}\left\{\hat{p}_{1}, \ldots \hat{p}_{n}\right\}$ by

$$
\widehat{P}_{n} u=\sum_{i, j=1}^{n}\left\langle u, \widetilde{\widetilde{A}} \hat{p}_{i}\right\rangle \hat{b}_{i j} \hat{p}_{j}, \quad u \in \mathfrak{H},
$$

where $\left[\hat{b}_{i j}\right]$ is the matrix inverse to $\left[\left\langle\hat{p}_{i}, \widetilde{\widetilde{A}} \hat{p}_{j}\right\rangle\right]$. Then $\widehat{P}_{n}$ is an orthogonal projection with respect to the inner product induced by $\tilde{\tilde{a}}(u)$ defined so that for each $n$,

$$
\mathscr{R}\left(I-\widehat{P}_{n}\right)=\mathscr{R}\left(I-P_{n}\right) \text { and } \widetilde{\widetilde{A}} \widehat{\mathscr{P}}_{n}=\tilde{A} \mathscr{P}_{n} .
$$

Moreover, $\tilde{\tilde{a}}\left(\widehat{P}_{n} u\right) \leq \tilde{a}\left(P_{n} u\right)$ for all $u \in \mathfrak{H}(\mathrm{cf} .[11,19])$. Hence, the intermediate operators

$$
A_{n}^{\prime \prime}=A_{0}^{(\gamma)}+\widetilde{\widetilde{A}} \widehat{P}_{n}
$$

yield eigenvalues $\lambda_{i}\left(A_{n}^{\prime \prime}\right)$ which for each $i$ with $\lambda_{i}(A)<\gamma$ satisfy $\lambda_{i}\left(A_{n}^{\prime \prime}\right) \leq$ $\lambda_{i}\left(A_{n}\right) \leq \lambda_{i}(A)$, and $\lambda_{i}\left(A_{n}^{\prime \prime}\right) \rightarrow \lambda_{i}\left(A^{(\mu)}\right)=\lambda_{i}(A)$ as $n \rightarrow \infty$.

Now, since $A^{(\mu)}$ and $\widetilde{A}=A^{(\mu)}-A_{0}^{(\gamma)}$ are bounded, the preceding proof for the case of bounded operators yields a constant $K$ independent of $n$ such that

$$
\left|\lambda_{j}\left(A^{(\mu)}\right)-\lambda_{j}\left(A_{n}^{\prime \prime}\right)\right| \leq K\left\{\delta_{\mathscr{M}}^{2}\left(\widetilde{\widetilde{A}} \widehat{\mathscr{P}}_{n}\right)+\delta_{\mathscr{N}}^{2}\left(\widetilde{\widetilde{A}} \widehat{\mathscr{P}}_{n}\right)\right\}
$$

where $\mathscr{M}=\mathscr{U}^{\gamma}$ and $\mathscr{N}=\mathscr{S}+\mathscr{U}_{0}^{\gamma}$. The proof is now complete, since $\lambda_{j}\left(A^{(\mu)}\right)=\lambda_{j}(A) \geq \lambda_{j}\left(A_{n}\right) \geq \lambda_{j}\left(A_{n}^{\prime \prime}\right)$ and, by construction, $\widetilde{\widetilde{A}} \widehat{\mathscr{P}}_{n}=\tilde{A} \mathscr{P}_{n}$.

Since the proof of Theorem 3.1 actually proceeds by estimating the differences between the unknown eigenvalues and eigenvectors of the auxiliary operator $A_{n}^{\prime}$ and those of $A$, it fails to provide useful estimates for the eigenvectors or eigenprojections of $A_{n}$. Our next theorem provides such estimates, but yields eigenvalue estimates which are in practice not as good as those of Theorem 3.1.

Theorem 3.2. Let $\lambda_{h}(A)=\lambda_{h+1}(A)=\cdots=\lambda_{h+m-1}(A)$ be an eigenvalue of $A$ below $\gamma$ with multiplicity $m$ and corresponding eigenspace $\mathscr{S}$. Let $P$ be the orthogonal projection onto $\mathscr{S}$, and let $\widetilde{P}$ be the projection onto $\mathscr{S}$ which is orthogonal with respect to the inner product induced by ã. Further assume that the convergence criteria of Theorem 2.1 or of Theorem 2.5 are satisfied and that 
$\left\|P_{n}^{*}\right\| \delta_{\mathscr{N}}\left(\widetilde{A} \mathscr{P}_{n}\right) \rightarrow 0$ as $n \rightarrow \infty$, where $\mathcal{N}=\mathscr{S}+\mathscr{U}_{0}^{\gamma}$. Then for each $j=h$, $h+1, \ldots, h+m-1$, and all $n$ large enough,

$$
\left|\lambda_{j}(A)-\lambda_{j}\left(A_{n}\right)\right| \leq K\left\{\left\|\tilde{A}^{1 / 2}\left(I-P_{n}\right) \tilde{P}\right\|^{2}+\left\|P_{n}^{*}\right\|^{2} \delta_{\mathscr{N}}^{2}\left(\tilde{A}_{n}\right)\right\}
$$

and

$$
\left\|R_{n} P-P\right\| \leq K\left\|P_{n}^{*}\right\| \delta_{\mathscr{N}}\left(\widetilde{A} \mathscr{P}_{n}\right),
$$

where $R_{n}$ is the orthogonal projection onto the $m$-dimensional space spanned by the eigenvectors of $A_{n}$ corresponding to the eigenvalues $\left\{\lambda_{j}\left(A_{n}\right)\right\}_{j=h}^{h+m-1}$. In particular, if $m=1, n$ is large enough, and the eigenvector $u_{h}^{[n]}$ of $A_{n}$ corresponding to $\lambda_{h}\left(A_{n}\right)$ is normalized by $\left\|u_{h}^{[n]}\right\|=1$ and $\left\langle u_{h}^{[n]}, u_{h}\right\rangle \geq 0$,

$$
\left\|u_{h}^{[n]}-u_{h}\right\| \leq K\left\|P_{n}^{*}\right\| \delta_{\mathscr{N}}\left(\tilde{A} \mathscr{P}_{n}\right)
$$

Herein, $K$ is a generic constant independent of $n$.

Proof. We use Theorem 2.6 with $S=A_{n},(c, d)$ any isolating interval for $\lambda_{h}(A)$, and $\left\{v_{k}\right\}_{k=1}^{m}$ an orthonormal basis for the eigenspace $\mathscr{S}$ which also diagonalizes the $m \times m$ matrix $\left[\left\langle A_{n} v_{k}, v_{l}\right\rangle\right]$, yielding eigenvalues $\eta_{1} \leq \eta_{2} \leq$ $\cdots \leq \eta_{m}$ given by $\eta_{k}=\left\langle A_{n} v_{k}, v_{k}\right\rangle, k=1, \cdots, m$. Then

$$
\begin{aligned}
\eta_{k} & =\left\langle A v_{k}, v_{k}\right\rangle+\left\langle\left(A_{n}-A\right) v_{k}, v_{k}\right\rangle \\
& =\lambda_{h}(A)+\left\langle\widetilde{A}\left(P_{n}-I\right) v_{k}, v_{k}\right\rangle=\lambda_{h}(A)+\tilde{a}\left(\left(P_{n}-I\right) v_{k}\right),
\end{aligned}
$$

so that

and

$$
\left|\eta_{k}-\lambda_{h}(A)\right| \leq\left\|\widetilde{A}^{1 / 2}\left(P_{n}-I\right) \widetilde{P}\right\|^{2}
$$

$$
\begin{aligned}
\theta_{k} & =\left\|\left(A_{n}-\eta_{k}\right) v_{k}\right\|=\left\|\left[\tilde{A}\left(P_{n}-I\right)-\left\langle\tilde{A}\left(P_{n}-I\right) v_{k}, v_{k}\right\rangle\right] v_{k}\right\| \\
& \leq\left\|\tilde{A}\left(P_{n}-I\right) v_{k}\right\| .
\end{aligned}
$$

Thus, since $\tilde{A}\left(P_{n}-I\right)$ is selfadjoint,

$$
\begin{aligned}
\theta_{k} & \leq\left\|\left(P_{n}^{*}-I\right) \tilde{A} v_{k}\right\|=\left\|\left(P_{n}^{*}-I\right)\left(I-Q_{n}\right) \tilde{A} v_{k}\right\| \\
& \leq\left(\left\|P_{n}^{*}\right\|+1\right)\left\|\left(I-Q_{n}\right) \tilde{A} v_{k}\right\|,
\end{aligned}
$$

where $Q_{n}$ is the orthogonal projection onto $\mathscr{R}\left(P_{n}^{*}\right)=\widetilde{A} \mathscr{P}_{n}$. Now, $\left\|P_{n}^{*}\right\| \geq 1$, $\widetilde{A} v_{k}=\left(\lambda_{h}(A)-A_{0}^{(\gamma)}\right) v_{k} \in \mathcal{N}$, and $\left\|\tilde{A} v_{k}\right\| \leq \gamma-\lambda_{1}\left(A_{0}\right)$, so

$$
\theta_{k} \leq 2\left\|P_{n}^{*}\right\|\left(\gamma-\lambda_{1}\left(A_{0}\right)\right) \delta_{\mathscr{N}}\left(\tilde{A} \mathscr{P}_{n}\right)
$$

By hypothesis, $\theta_{k} \rightarrow 0$ and $\eta_{k} \rightarrow \lambda_{h}(A)$ as $n \rightarrow \infty$, so Theorem 2.6 implies the conclusions of the theorem.

\section{Applications}

In order to apply the preceding estimates to differential eigenvalue problems, it is convenient to dominate the containment gaps of Theorems 3.1 and 3.2 in terms of spectral projections of an auxiliary operator $B$. Specifically, let $B$ be a positive definite selfadjoint operator in $\mathfrak{H}$ such that $\mathscr{D}(B) \subset \mathscr{D}(\tilde{A})$ and $\|\tilde{A} u\| \leq \beta\|B u\|, \beta>0$, for all $u \in \mathscr{D}(B)$, with $B^{-1}$ compact. Let

$$
0<\mu_{1} \leq \mu_{2} \leq \cdots \leq \mu_{i} \leq \cdots \nearrow \infty
$$


be the eigenvalues of $B$ enumerated as usual according to multiplicity, with corresponding eigenvectors $\left\{p_{i}\right\}$ orthonormal in $\mathfrak{H}$. If these vectors $\left\{p_{i}\right\}$ are employed as the trial vectors to construct the projection operators $\left\{P_{n}\right\}$ of $(2.1)$, then the following estimate is obtained in [11].

Theorem 4.1. If for all eigenvalues $\lambda_{i}(A)$ and $\lambda_{i}\left(A_{0}\right)$ less than $\gamma$, both $u_{i}$ and $(A-\gamma)^{-1} u_{i}^{0}$ are in $\mathscr{D}\left(B^{\tau}\right)$ with $\tau>1$, then

$$
\delta_{\mathscr{g}}\left(\tilde{A}_{n}\right)=o\left(\mu_{n+1}^{1-\tau}\right) \quad \text { as } n \rightarrow \infty,
$$

where $\mathcal{J}=\mathscr{U}^{\gamma}+\mathscr{U}_{0}^{\gamma}$.

Herein, $O$ is the usual Landau symbol, $B^{\tau}$ denotes the unique positive definite $\tau$ th power of $B$, and we assume without loss of generality that $\gamma$ is in the resolvent set of $A$. Since both $\mathscr{U}^{\gamma}$ and $\mathscr{S}+\mathscr{U}_{0}^{\gamma}$ are contained in $\mathscr{U}^{\gamma}+\mathscr{U}_{0}^{\gamma}$, this provides a technique for implementing Theorem 3.1.

A closely related, but simpler, analysis than that leading to (4.1) yields

$$
\left\|\widetilde{A}^{1 / 2}\left(I-P_{n}\right) \widetilde{P}\right\|^{2}=o\left(\mu_{n+1}^{1-2 \tau}\right) \quad \text { as } n \rightarrow \infty,
$$

under slightly weaker hypotheses than those of Theorem 4.1. This reveals that as long as (4.1) is used to estimate $\delta_{\mathscr{N}}\left(\widetilde{A}_{\mathscr{P}_{n}}\right), \mathscr{N}=\mathscr{S}+\mathscr{U}_{0}^{\gamma}$, the eigenvalue estimate of Theorem 3.2 can only be as good asymptotically as that of Theorem 3.1 if $\left\{\left\|P_{n}^{*}\right\|\right\}$ is bounded, unless $\left\|P_{n}^{*}\right\| \delta_{\mathscr{N}}\left(\widetilde{A} P_{n}\right)=\mathscr{O}\left(\delta_{\mathscr{M}}\left(\widetilde{A} P_{n}\right)\right)$ as $n \rightarrow \infty$. We now estimate $\left\|P_{n}^{*}\right\|$ in terms of $\mu_{n}$, so that Theorem 3.2 may be applied to obtain eigenvector and eigenprojection estimates in differential problems.

Theorem 4.2. One has $\left\|P_{n}\right\|=\left\|P_{n}^{*}\right\| \leq(\beta / \alpha) \mu_{n}$. Further, let $b(u)$ be the closure of the quadratic form $\langle B u, u\rangle$. If also $\mathscr{D}(\tilde{a}) \subset \mathscr{D}(b)$, and there exists $\rho>0$ such that $\rho b(u) \leq \tilde{a}(u)$ for all $u \in \mathscr{D}(\tilde{a})$, then

$$
\left\|P_{n}^{*}\right\| \leq(\beta / \rho)\left(\mu_{n} \sum_{i=1}^{n} \mu_{i}^{-1}\right)^{1 / 2} .
$$

Proof. Recall that $B p_{i}=\mu_{i} p_{i}$, and take $\left\langle p_{i}, p_{j}\right\rangle=\delta_{i j}$. Further select vectors $q_{1}, \ldots, q_{n}$ satisfying $\operatorname{span}\left\{q_{1}, \ldots, q_{n}\right\}=\operatorname{span}\left\{p_{1}, \ldots, p_{n}\right\}=\mathscr{P}_{n},\left\langle q_{i}, q_{j}\right\rangle=$ $\delta_{i j}, \tilde{a}\left(q_{i}, q_{j}\right)=\eta_{i} \delta_{i j}$ with $\eta_{1} \leq \eta_{2} \leq \cdots \leq \eta_{n}$. Then

$$
P_{n} u=\sum_{i=1}^{n} \frac{\left\langle u, \tilde{A} q_{i}\right\rangle}{\eta_{i}} q_{i},
$$

so that

$$
P_{n}^{*} u=\sum_{i=1}^{n} \frac{\left\langle u, q_{i}\right\rangle}{\eta_{i}} \tilde{A} q_{i}
$$

Define $\sigma_{1}, \cdots, \sigma_{n}$ by

$$
\sum_{i=1}^{n} \frac{\left\langle u, q_{i}\right\rangle}{\eta_{i}} q_{i}=\sum_{i=1}^{n} \sigma_{i} p_{i} .
$$

Then,

$$
P_{n}^{*} u=\widetilde{A} B^{-1}\left(\sum_{i=1}^{n} \sigma_{i} \mu_{i} p_{i}\right),
$$


and so,

$$
\begin{aligned}
\left\|P_{n}^{*} u\right\|^{2} & \leq\left\|\widetilde{A} B^{-1}\right\|^{2} \sum_{i=1}^{n} \sigma_{i}^{2} \mu_{i}^{2} \leq \beta^{2} \mu_{n}^{2} \sum_{i=1}^{n} \sigma_{i}^{2} \\
& =\beta^{2} \mu_{n}^{2} \sum_{i=1}^{n} \frac{\left|\left\langle u, q_{i}\right\rangle\right|^{2}}{\eta_{i}^{2}} \leq(\beta / \alpha)^{2} \mu_{n}^{2}\|u\|^{2},
\end{aligned}
$$

i.e., $\left\|P_{n}^{*}\right\| \leq(\beta / \alpha) \mu_{n}$.

To obtain (4.2), first note that, if also $\rho b(u) \leq \tilde{a}(u)$, then

$$
\sum_{i=1}^{n} \sigma_{i}^{2} \mu_{i}=b\left(\sum_{i=1}^{n} \sigma_{i} p_{i}\right) \leq \rho^{-1} \tilde{a}\left(\sum_{i=1}^{n} \frac{\left\langle u, q_{i}\right\rangle}{\eta_{i}} q_{i}\right)=\rho^{-1} \sum_{i=1}^{n} \frac{\left|\left\langle u, q_{i}\right\rangle\right|^{2}}{\eta_{i}} .
$$

Now $\left\{\eta_{i}\right\}$ are the Rayleigh-Ritz eigenvalues of $\tilde{A}$ corresponding to the subspace $\mathscr{P}_{n}$. Since $\rho b(u) \leq \tilde{a}(u)$, these dominate the Rayleigh-Ritz eigenvalues of $\rho B$ corresponding to the subspace $\mathscr{P}_{n}$. Thus, $\rho \mu_{i} \leq \eta_{i}, i=1, \ldots, n$. So,

$$
\sum_{i=1}^{n} \sigma_{i}^{2} \mu_{i} \leq \rho^{-2}\left(\sum_{i=1}^{n} \mu_{i}^{-1}\right)\|u\|^{2}
$$

Hence,

$$
\begin{aligned}
\left\|P_{n}^{*} u\right\|^{2} & \leq\left\|\widetilde{A} B^{-1}\right\|^{2} \sum_{i=1}^{n} \sigma_{i}^{2} \mu_{i}^{2} \leq \beta^{2} \mu_{n} \sum_{i=1}^{n} \sigma_{i}^{2} \mu_{i} \\
& \leq(\beta / \rho)^{2} \mu_{n}\left(\sum_{i=1}^{n} \mu_{i}^{-1}\right)\|u\|^{2}
\end{aligned}
$$

which completes the proof.

\section{EXAMPLES}

Two examples of convergence rates for differential eigenvalue problems, with one involving nontrivial essential spectrum, were given previously in [11]. For those examples, Theorem 3.1 squares the convergence rate estimates of [11] for eigenvalues, and Theorem 3.2 provides rate of convergence estimates for eigenvectors and eigenprojections. We present here additional applications to Sturm-Liouville problems, which allow direct comparison of our results with those of $[12,15]$ for the method of special choice.

Let $\mathfrak{H}=L^{2}(0,1)$ and, for $u \in H_{0}^{1}(0,1)$-the closure of $C_{0}^{\infty}(0,1)$ in the Sobolev space $H^{1}(0,1)$-let

$$
a(u)=\int_{0}^{1}\left(\left|u^{\prime}\right|^{2}+q|u|^{2}\right) d x
$$

where $q$ is a nonnegative function in $C^{3}(0,1)$. Then $\mathscr{D}(A)=H^{2}(0,1) \cap$ $H_{0}^{1}(0,1)$, and the eigenvalue problem for $A$ means

$$
A u=-u^{\prime \prime}+q u=\lambda u \quad \text { in }(0,1), \quad u(0)=u(1)=0 .
$$

Let

$$
a_{0}(u)=\int_{0}^{1}\left|u^{\prime}\right|^{2} d x, \quad u \in H_{0}^{1}(0,1)
$$


i.e.,

$$
A_{0} u=-u^{\prime \prime} \quad \text { in }(0,1), \quad u(0)=u(1)=0 .
$$

If we take $B=A_{0}$, so that $p_{k}=u_{k}^{0}$ and $\mu_{k}=\lambda_{k}^{0}=k^{2} \pi^{2}$, both (4.1) and (4.2) apply. To find $\tau$ in (4.1), first note that with $\lambda_{j} \equiv \lambda_{j}(A)$,

$$
B u_{j}=A_{0} u_{j}=\lambda_{j} u_{j}-q u_{j} \in H^{2}(0,1) \cap H_{0}^{1}(0,1),
$$

and so

$$
A_{0}^{2} u_{j}=\left(\lambda_{j}^{2}-2 \lambda_{j} q+q^{2}+q^{\prime \prime}\right) u_{j}+2 q^{\prime} u_{j}^{\prime} \in H^{1}(0,1)
$$

so that $u_{j} \in \mathscr{D}\left(B^{\tau}\right)=\mathscr{D}\left(A_{0}^{\tau}\right)$ for all $\tau<9 / 4$ (cf. either of $[17,20]$ ). In addition,

$$
A_{0}(A-\gamma)^{-1} u_{j}^{0}=u_{j}^{0}+(\gamma-q)(A-\gamma)^{-1} u_{j}^{0} \in H^{2}(0,1) \cap H_{0}^{1}(0,1),
$$

and

$$
\begin{aligned}
A_{0}^{2}(A-\gamma)^{-1} u_{j}^{0}= & \left(\lambda_{j}+\gamma-q\right) u_{j}^{0}+q^{\prime \prime}(A-\gamma)^{-1} u_{j}^{0} \\
& +2 q^{\prime}\left[(A-\gamma)^{-1} u_{j}^{0}\right]^{\prime}+(\gamma-q)^{2}(A-\gamma)^{-1} u_{j}^{0} \in H^{1}(0,1),
\end{aligned}
$$

so that $(A-\gamma)^{-1} u_{j}^{0} \in \mathscr{D}\left(A_{0}^{\tau}\right)$ for all $\tau<9 / 4$. Thus, since $\mu_{n}=\lambda_{n}^{0}=n^{2} \pi^{2}$, Theorems 3.1 and 4.1 yield

$$
\left|\lambda_{j}(A)-\lambda_{j}\left(A_{n}\right)\right|=o\left(n^{-\delta}\right) \quad \text { as } n \rightarrow \infty \text { for all } \delta<5
$$

If we were to integrate by parts on the expressions for $A_{0}^{2} u_{j}$ and $A_{0}^{2}(A-\gamma)^{-1} u_{j}^{0}$ as in [12], we could improve this estimate to $O\left(n^{-5}\right)$ as $n \rightarrow \infty$, which is the convergence rate given in $[12,15]$ for the method of special choice. Since the eigenvalues of $A$ are simple, Theorem 3.2, Theorem 4.1, and (conclusion (4.2) of) Theorem 4.2 now give the eigenvector estimate

$$
\left\|u_{i}^{[n]}-u_{i}\right\|=o\left(n^{-\delta}\right) \quad \text { as } n \rightarrow \infty \text { for all } \delta<3 / 2,
$$

which would improve to $O\left(n^{-3 / 2}\right)$ by use of integration by parts in the fashion mentioned above. The special choice rate of $[12,15]$ is $O\left(n^{-5 / 2}\right)$.

In order to illustrate the derived eigenvalue rate in this setting, we consider the parabolic cylinder equation, which is obtained by setting $q(x)=x^{2}$ above. Intermediate problems are constructed as described in $\S 2$, using base problem eigenfunctions to define the projection subspaces $\mathscr{P}_{n}=\operatorname{span}\left\{u_{1}^{0}, u_{2}^{0}, \ldots, u_{n}^{0}\right\}$. The value of $\gamma$ is fixed at 90.0 , leaving three base problem eigenvalues below the truncation point. Resolution of the resulting intermediate problem of order $n$ requires the solution of a generalized matrix eigenvalue problem of dimension $3+n$ given by

$$
\left[\begin{array}{ll}
\mathbf{F} & \mathbf{G}^{t} \\
\mathbf{G} & \mathbf{H}
\end{array}\right]\left[\begin{array}{l}
x_{1} \\
x_{2}
\end{array}\right]=(\lambda-\gamma)\left[\begin{array}{cc}
\mathbf{R} & 0 \\
0 & \mathbf{S}
\end{array}\right]\left[\begin{array}{l}
x_{1} \\
x_{2}
\end{array}\right],
$$

where $\mathbf{R}=\operatorname{diag}\left\{\left\|u_{i}^{0}\right\|^{2} /\left(\lambda_{i}^{0}-\gamma\right)\right\}, \quad \mathbf{S}=\left[\left\langle p_{k}, \tilde{A} p_{l}\right\rangle\right], \quad \mathbf{F}=\operatorname{diag}\left\{\left\|u_{i}^{0}\right\|^{2}\right\}, \quad \mathbf{G}=$ $\left[\left\langle u_{i}^{0}, \tilde{A} p_{l}\right\rangle\right]$, and $\mathbf{H}=\left[\left\langle\widetilde{A} p_{k}, \widetilde{A} p_{l}\right\rangle\right]$. Calculations were performed on a Sun $3 / 60$ workstation in double precision (unit roundoff $\approx 1.1 \times 10^{-16}$ ). The QZ method [24] was used to solve the matrix eigenvalue problem (5.1). Matrix eigenvalues associated with bounds were computed to an estimated relative accuracy exceeding $10^{-12}$. A selection of results is listed in Table 1 and summarized graphically in Figure 1. Complementary upper bounds were found with a 
TABLE 1. Parabolic cylinder equation

\begin{tabular}{|c|c|c|c|c|c|}
\hline Index & $\begin{array}{c}\text { Base } \\
\text { Problem }\end{array}$ & $\begin{array}{l}\quad \text { Intermedi } \\
n=5\end{array}$ & $\begin{array}{l}\begin{array}{l}\text { Problem } \\
n=10\end{array}\end{array}$ & $\begin{array}{l}\text { Size } \\
\quad n=20\end{array}$ & $\begin{array}{l}\text { Rayleigh-Ritz } \\
\quad(n=40)\end{array}$ \\
\hline 1 & 9.8696 & 10.151163761501 & 10.151164018432 & 10.151164030016 & 10.151164030453 \\
\hline 2 & 39.478 & 39.799390484344 & 39.799392917539 & 39.799393000748 & 39.799393003660 \\
\hline 3 & 88.826 & 89.153654454880 & 89.154328758500 & 89.154342048421 & 89.154342456268 \\
\hline
\end{tabular}

Rayleigh-Ritz calculation of order 40 , using base problem eigenfunctions as trial functions. In order to gauge rates of convergence, Figure 1 presents eigenvalue bracket widths plotted against intermediate problem order $n$, on a log-log scale. Parallel linear asymptotes are evident in each case, with calculated slopes lying between 4.95 and 4.98 . This is within a $1 \%$ deviation from the theoretically predicted asymptotic slope of 5 .

If, for the general Sturm-Liouville problem above, the potential $q(x) \epsilon$ $C^{3}(0,1)$ also satisfies the additional condition $q^{\prime}(0)=q^{\prime}(\pi)=0$, then $A_{0}^{2} u_{j}$ and $A_{0}^{2}(A-\gamma)^{-1} u_{j}^{0}$ are in $H_{0}^{1}(0,1)$, so that $u_{j}$ and $(A-\gamma)^{-1} u_{j}^{0}$ are in $\mathscr{D}\left(A_{0}^{5 / 2}\right)$. Hence,

$$
\left|\lambda_{i}(A)-\lambda_{i}\left(A_{n}\right)\right|=o\left(n^{-6}\right) \text { as } n \rightarrow \infty,
$$

and

$$
\left\|u_{i}^{[n]}-u_{i}\right\|=o\left(n^{-2}\right) \text { as } n \rightarrow \infty .
$$

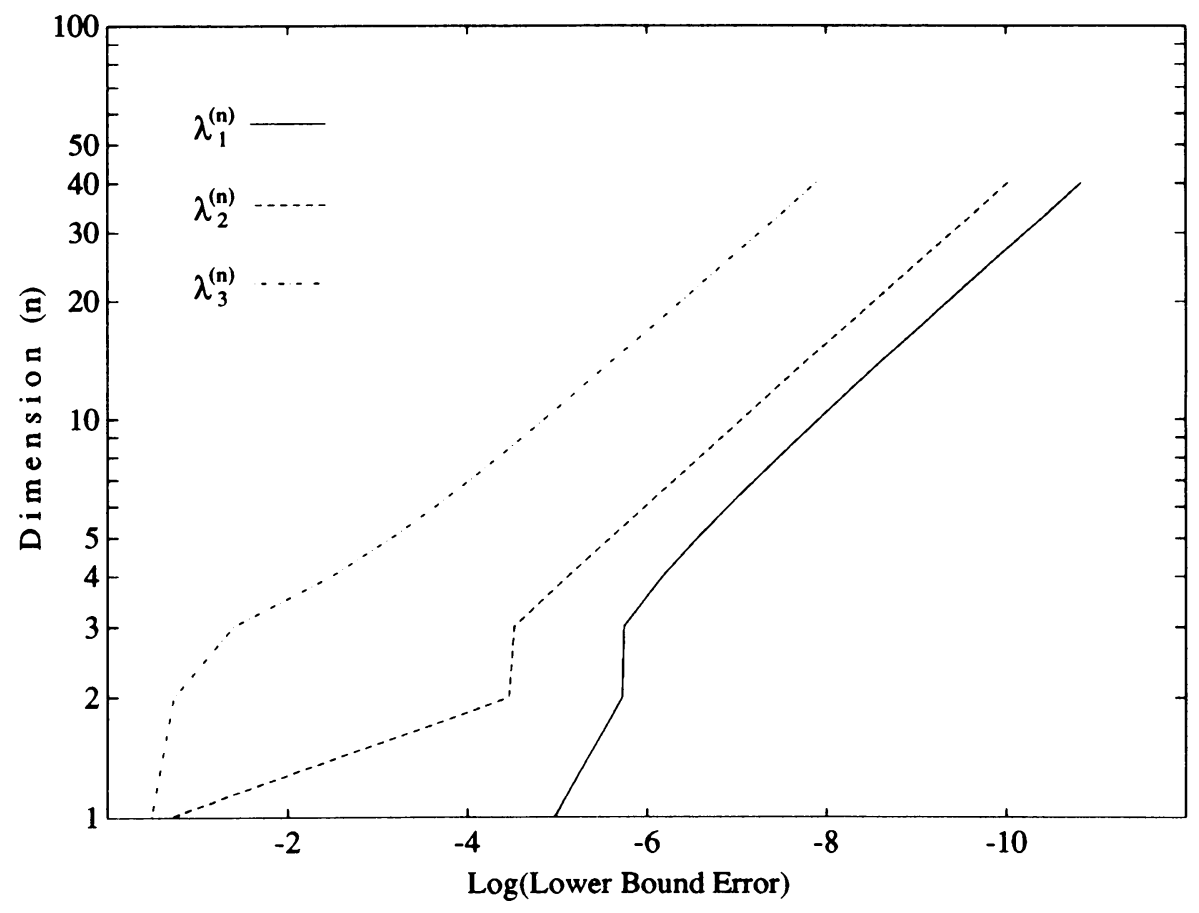

FIGURE 1. Parabolic cylinder equation 
More rapid rates of convergence follow from additional smoothness of $q$ and the vanishing of additional derivatives of $q$ at 0 and 1 .

Still more rapid rates occur if we were to consider periodic boundary conditions in the preceding. That is, let $\mathfrak{H}=L^{2}(0,1)$, let $q$ be the restriction to $(0,1)$ of a nonnegative periodic $C^{\infty}$ function with period 1 , and for $u \in\left\{v \in H^{1}(0,1): v(0)=v(1)\right\}$ let

$$
a(u)=\int_{0}^{1}\left(\left|u^{\prime}\right|^{2}+q|u|^{2}\right) d x .
$$

This means

$$
A u=-u^{\prime \prime}+q u \quad \text { in }(0,1), u(0)=u(1), u^{\prime}(0)=u^{\prime}(1)
$$

Take

$$
a_{0}(u)=\int_{0}^{1}\left|u^{\prime}\right|^{2} d x, \quad u \in \mathscr{D}\left(a_{0}\right)=\mathscr{D}(a)
$$

which means

$$
A_{0} u=-u^{\prime \prime} \quad \text { in }(0,1), u(0)=u(1), u^{\prime}(0)=u^{\prime}(1) .
$$

The methods of the preceding example may be followed to obtain an "infinite order of convergence" for the eigenvalues and eigenprojections, i.e., $o\left(n^{-\delta}\right)$ for every $\delta>0$. These conclusions are the same as for the method of special choice $[12,15]$. This rapid estimate for the eigenvalues also follows from the estimate of [11].

As a final example, for $u \in H^{1}(\mathbb{R})$ let

$$
a(u)=\int_{-\infty}^{\infty}\left(\left|u^{\prime}\right|^{2}+q|u|^{2}\right) d x
$$

where $q \in C^{\infty}(\mathbb{R}) \cap L^{2}(\mathbb{R})$ is even with $q(0)<0, q$ is strictly increasing on $\left[0, x_{1}\right]$ to a positive maximum at $x_{1}$, and $q$ is decreasing on $\left[x_{1}, \infty\right)$ with $q$ and all derivatives of $q$ tending to zero at infinity. Some of the conditions on $q$ can be relaxed in the following, but these hypotheses are consistent with typical potential well considerations in quantum mechanics and provide for ease of exposition. Then, with $\mathfrak{H}=L^{2}(\mathbb{R}), \mathscr{D}(A)=H^{2}(\mathbb{R})$, the eigenvalue problem for $A$ means

$$
-u^{\prime \prime}+q u=\lambda u \quad \text { on } \mathbb{R}, u \in L^{2}(\mathbb{R})
$$

Let $x_{0}$ be the unique zero of $q$ in $\left(0, x_{1}\right)$. An explicitly solvable lower bound problem is obtained from

$$
-u^{\prime \prime}+q_{0} u=\lambda u \quad \text { on } \mathbb{R}, u \in L^{2}(\mathbb{R}),
$$

with $q_{0}$ the "square well" potential:

$$
q_{0}(x)= \begin{cases}q(0)+\gamma, & -x_{0} \leq x \leq x_{0}, \\ \gamma, & |x|>x_{0} .\end{cases}
$$

Herein, $\gamma<0$ is great enough that all negative eigenvalues of $A$ are less than $\gamma$. The negative number $\gamma$ will, as previously, be our truncation point-and has been added to the usual square well potential so that $\hat{a}(u)$, and therefore $\tilde{a}(u)$, 
are positive definite. This eigenvalue problem is obtained from the quadratic form,

$$
a_{0}(u)=\int_{-\infty}^{\infty}\left(\left|u^{\prime}\right|^{2}+q_{0}|u|^{2}\right) d x, \quad u \in H^{1}(\mathbb{R}),
$$

and $\mathscr{D}\left(A_{0}\right)=\mathscr{D}(A)=H^{2}(\mathbb{R})$.

To implement Theorem 4.1, let $B$ be the harmonic oscillator, i.e.,

$$
B=\frac{d^{2}}{d x^{2}}+x^{2}
$$

with $\mathscr{D}(B)=H^{2}(\mathbb{R}) \cap \mathscr{D}\left(x^{2}\right)$, where, as usual,

$$
\mathscr{D}\left(x^{2}\right)=\left\{u \in L^{2}(\mathbb{R}): \int_{-\infty}^{\infty} x^{4}|u(x)|^{2} d x<\infty\right\} .
$$

Then $B$ is selfadjoint on $\mathscr{D}(B)$ (cf. [28]), and $\mu_{k}=2 k-1$. An analysis like that of the preceding examples and pp. 225-226 of [11] yields $u_{j} \in \mathscr{D}\left(B^{\tau}\right)$ for all $\tau>0$, but only $(A-\gamma)^{-1} u_{j}^{0} \in \mathscr{D}\left(B^{\tau}\right)$ for all $\tau<9 / 4$, owing to the jumps in $q_{0}$ at $\pm x_{0}$. On the other hand, if $q_{0}$ was in $C_{0}^{\infty}(\mathbb{R})$, we would have $(A-\gamma)^{-1} u_{j}^{0}$ in $\mathscr{D}\left(B^{\tau}\right)$ for all $\tau>0$, and thus an infinite-order convergence, but the spectral problem for the base operator would not be explicitly solvable. We wish now to show that Lemma 3.5 of [10] can be used to convert this observation into a proof of an infinite order of convergence of the eigenvalues, starting from the square well base problem.

So let $V_{0} \in C^{\infty}(\mathbb{R})$ be such that $V_{0}-\gamma \in C_{0}^{\infty}(\mathbb{R})$ and $V_{0} \leq q_{0}$. Further, let $\mathbb{A}_{0}$ denote the one-dimensional Schrödinger operator corresponding to $V_{0}$. By regularization, such $V_{0}$ may be constructed to approximate $q_{0}$ within any desired tolerance in $L^{2}(\mathbb{R})$ (cf. [21]). Then, since

$$
\int_{-\infty}^{\infty}\left|\left(q_{0}-V_{0}\right) u\right|^{2} d x \leq\|u\|_{L^{\infty}(\mathbb{R})}^{2} \cdot\left\|q_{0}-V_{0}\right\|_{L^{2}(\mathbb{R})}^{2} \leq\|u\|_{H^{1}(\mathbb{R})}^{2} \cdot\left\|q_{0}-V_{0}\right\|_{L^{2}(\mathbb{R})}^{2}
$$

for all $u \in H^{1}(\mathbb{R})$, it follows that $\mathbb{A}_{0}$ can be chosen to approximate $A_{0}$ arbitrarily closely in the norm resolvent sense (cf. [27]). Thus, we may assume that $\mathbb{A}_{0}$ and $A_{0}$ have the same number of eigenvalues below $\gamma$, and then, given $\varepsilon>0$, there exists such a $V_{0}$ satisfying

$$
\begin{aligned}
& \left\|A_{0}^{(\gamma)} u-\mathbb{A}_{0}^{(\gamma)} u\right\|=\| \sum\left[\lambda_{i}\left(A_{0}\right)-\gamma\right]\left\langle u, u_{i}\left(A_{0}\right)\right\rangle u_{i}\left(A_{0}\right) \\
& \quad-\sum\left[\lambda_{i}\left(\mathbb{A}_{0}\right)-\gamma\right]\left\langle u, u_{i}\left(\mathbb{A}_{0}\right)\right\rangle u_{i}\left(\mathbb{A}_{0}\right) \| \\
& \leq\left\{2\left|\lambda_{1}\left(A_{0}\right)-\gamma\right|\left(\sum\left\|u_{i}\left(A_{0}\right)-u_{i}\left(\mathbb{A}_{0}\right)\right\|\right)+\sum\left(\lambda_{i}\left(A_{0}\right)-\lambda_{i}\left(\mathbb{A}_{0}\right)\right)\right\}\|u\| \\
& \leq \varepsilon\|u\| \quad \text { for all } u \in L^{2}(\mathbb{R}),
\end{aligned}
$$

where the summation is over all eigenvalues below $\gamma$. Then, for each $u \in \mathfrak{H}=$ $L^{2}(\mathbb{R})$, i.e.,

$$
\left\langle\mathbb{A}_{0}^{(\gamma)} u, u\right\rangle-\varepsilon\|u\|^{2} \leq\left\langle A_{0}^{(\gamma)} u, u\right\rangle
$$

$$
\left\langle\left(\mathbb{A}_{0}-\varepsilon\right)^{(\gamma-\varepsilon)} u, u\right\rangle \leq\left\langle A_{0}^{(\gamma)} u, u\right\rangle
$$

As noted above, the intermediate problem eigenvalues obtained from the truncated base operator $\left(A_{0}-\varepsilon\right)^{(\gamma-\varepsilon)}$ have an infinite order of convergence. But 
by Lemma 3.5 of [10], these give lower bounds for the intermediate problem eigenvalues obtained from the truncated base operator $A_{0}^{(\gamma)}$. Hence,

$$
\left|\lambda_{i}(A)-\lambda_{i}\left(A_{n}\right)\right|=o\left(n^{-\delta}\right) \text { as } n \rightarrow \infty \text { for all } \delta>0 .
$$

In order to illustrate this last result, consider the one-dimensional Schrödinger operator (5.2) with potential defined by

$$
q(x)=b\left(x^{2}-a^{2}\right) \exp \left(-c x^{2}\right)
$$

We take (5.3) as base operator, with $x_{0}=a$. For convenience, we restrict ourselves to the even symmetry class of functions solving (5.2) and (5.3). The lower spectrum of $A_{0}$ is resolvable and consists of simple eigenvalues that are solutions in $\lambda$ to

$$
\cot \left(a \sqrt{\lambda+\gamma+b a^{2}}\right)=\sqrt{-\left(1+\frac{b a^{2}}{\lambda+\gamma}\right)}
$$

lying in the interval $\left(\gamma-b a^{2}, \gamma\right)$. The lowest point of the essential spectrum of $A_{0}$ is given by $\gamma$, and the number of eigenvalues of $A_{0}$ smaller than $\gamma$ (represented herein as $M)$ is equal to the greatest integer smaller than $\left(a^{2} \sqrt{b} / \pi\right)+1$. These eigenvalues below $\gamma$ are labeled as before, $\lambda_{1}^{0}<\lambda_{1}^{0}<\cdots<\lambda_{M}^{0}$. The corresponding (unnormalized) eigenfunctions of $A_{0}$ are given by

$$
u_{i}^{0}= \begin{cases}\exp \left(-a \sqrt{\gamma-\lambda_{i}^{0}}\right) \cos \left(\sqrt{\lambda_{i}^{0}+b a^{2}-\gamma} x\right), \quad-a \leq x \leq a, \\ \cos \left(a \sqrt{\lambda_{i}^{0}+b a^{2}-\gamma}\right) \exp \left(-\sqrt{\gamma-\lambda_{i}^{0}}|x|\right) & \text { otherwise }\end{cases}
$$

Intermediate problems are then constructed as described in $\S 2$, using evenordered Hermite functions to define the projection subspaces $\mathscr{P}_{n}=$ $\operatorname{span}\left(p_{1}, p_{2}, \ldots, p_{n}\right)$ (i.e., $B p_{k}=(4 k-3) p_{k}$ for $\left.k=1,2, \ldots, n\right)$. Similar to what was done before, resolution of the resulting intermediate problem of order $n$ requires the resolution of a generalized matrix eigenvalue problem of dimension $M+n$, given by (5.1). ${ }^{1}$ In turn, these inner product matrices may be expressed in terms of the four basic inner product matrices: $\left[\left\langle u_{i}^{0}, p_{j}\right\rangle\right]$, $\left[\left\langle u_{i}^{0}, A p_{j}\right\rangle\right],\left[\left\langle A p_{i}, A p_{j}\right\rangle\right]$, and $\left[\left\langle A p_{i}, p_{j}\right\rangle\right]$. Analytical expressions may be obtained for $\left[\left\langle A p_{i}, p_{j}\right\rangle\right]$ and $\left[\left\langle A p_{i}, A p_{j}\right\rangle\right]$. However, inner products involving the base problem eigenvectors $\left\{u_{i}^{0}\right\}$ must be evaluated through quadrature. For reasons of economy and precision, the matrix elements $\left[\left\langle u_{i}^{0}, p_{j}\right\rangle\right]$ and $\left[\left\langle u_{i}^{0}, A p_{j}\right\rangle\right]$ are determined from recurrence relations that are derivable from the basic threeterm recurrence for Hermite polynomials. With this approach, transcendental function evaluations are reduced to the evaluation of the complementary error function, erfc $z$, and the quadrature of

$$
\int_{0}^{a} \cos \left(\sqrt{\lambda_{i}^{0}+b a^{2}-\gamma} x\right) \exp \left(-x^{2} / 2\right) d x
$$

for $i=1,2, \ldots, M$.

${ }^{1}$ Accurate calculation of the inner product matrices is a fairly involved undertaking in this case. The authors are grateful to $\mathrm{Mr}$. Gyou-Bong Lee for painstaking technical assistance in the computations described here. 
TABLE 2. Radial Schrödinger equation

$\begin{array}{cccccc}\text { Index } & \begin{array}{c}\text { Base } \\ \text { Problem }\end{array} & \begin{array}{c}\text { Intermediate } \\ n=5\end{array} & \begin{array}{c}\text { Problem } \\ n=10\end{array} & \begin{array}{c}\text { Size } \\ n=15\end{array} & \begin{array}{c}\text { Rayleigh-Ritz } \\ (n=20)\end{array} \\ 1 & -17.764 & -16.530730252641 & -16.530730006082 & -16.530730006081 & -16.530730006081 \\ 2 & -15.885 & -10.712190146655 & -10.711982838692 & -10.711982836444 & -10.711982836444 \\ 3 & -12.162 & -5.0350514466695 & -5.0116193956448 & -5.0116181724517 & -5.0116181724446 \\ 4 & -6.7072 & * & * & * & *\end{array}$

Script-size digits represent a contribution smaller than a posteriori estimates of accuracy.

* indicates a computed value above the lowest point of the essential spectrum.

Results are reported here for $a=3, b=2$, and $c=0.01$. Calculations were performed on a VAX 8800 in double precision carrying a unit roundoff $\approx 1.4 \times 10^{-17}$. Numerical quadratures were carried out in extended precision (mantissa length of 112 bits) using a globally adaptive 21 point/10 point Gauss-Kronrod scheme to an estimated relative accuracy of $10^{-14}$. As before, the matrix eigenvalue problem (5.1) was solved using the $Q Z$ method [24] with eigenvalues participating in bounds found to a relative accuracy of better than $10^{-11}$. An order-20 Rayleigh-Ritz calculation using even-ordered Hermite trial functions was performed to provide complementary upper bounds. Table 2 provides a sampling of these results for three intermediate problem orders. In Figure 2, the resulting eigenvalue bracket widths are plotted against intermediate

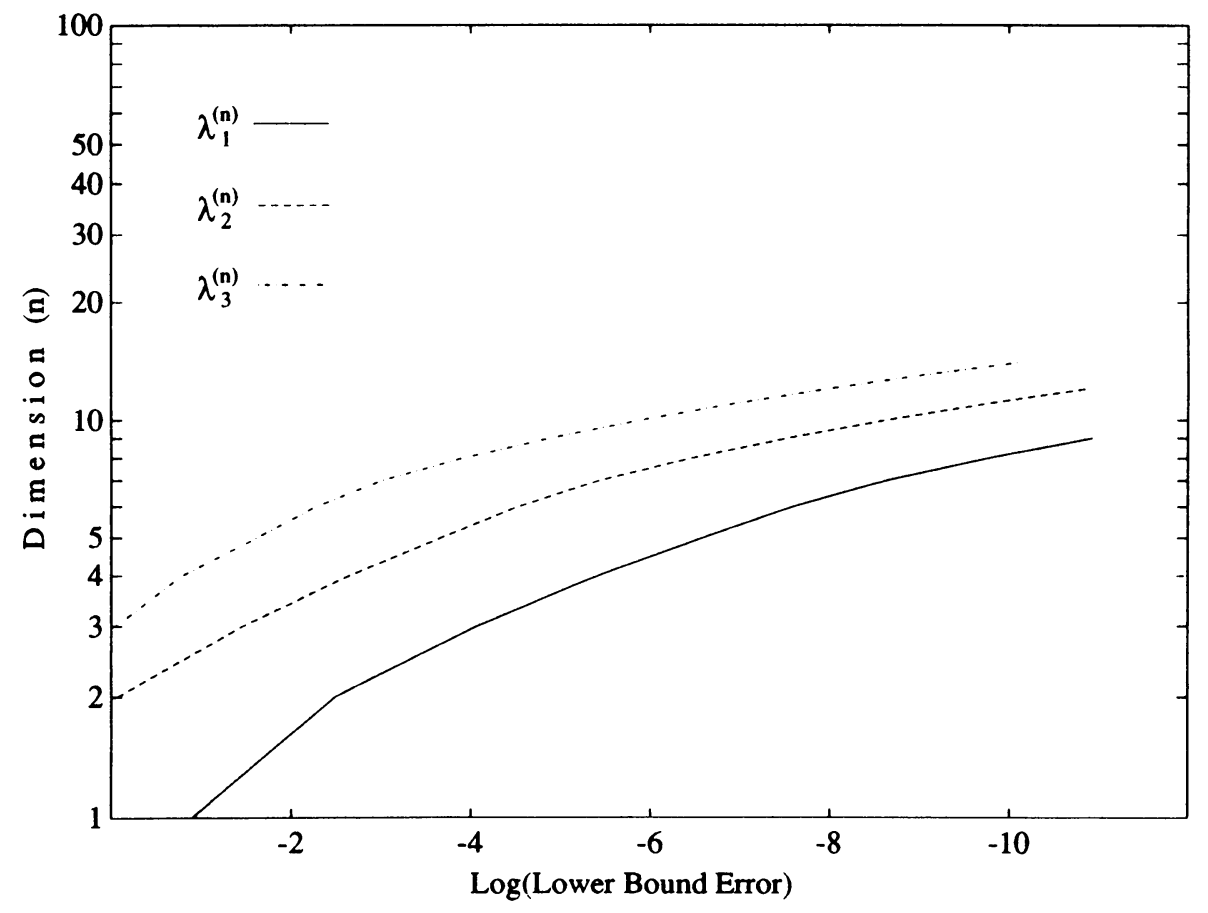

FIGURE 2. Radial Schrödinger equation 
problem order $n$, on a log-log scale. Notice that no linear asymptote is apparent for any of the three error curves-consistent with the predicted infinite order of convergence.

\section{BIBLIOGRAPHY}

1. N. Aronszajn, Approximation methods for eigenvalues of completely continuous symmetric operators, Proc. Sympos. on Spectral Theory and Differential Problems, Oklahoma A \& M College, Stillwater, 1951, pp. 179-202.

2. N. Aronszajn and A. Weinstein, Sur la convergence d'un procédé variationnel d'approximation dans la théorie des plaques encastrées, C. R. Acad. Sci. Paris 204 (1937), 96-98.

3. _ On the unified theory of eigenvalues of plates and membranes, Amer. J. Math. 64 (1942), 623-645.

4. N. W. Bazley, Lower bounds for eigenvalues, J. Math. Mech. 10 (1961), 289-303.

5. N. W. Bazley and D. W. Fox, Truncations in the method of intermediate problems for lower bounds to eigenvalues, J. Res. Nat. Bur. Standards 65B (1961), 105-111.

6. __ Error bounds for eigenvectors of self-adjoint operators, J. Res. Nat. Bur. Standards 66B (1962), 1-4.

7. Phys. 17 (1966), 1-37.

8. _ Comparison operators for lower bounds to eigenvalues, J. Reine Angew. Math. 223 (1966), 142-14.

9. C. Beattie, Some convergence results for intermediate problems with essential spectra, M.S.E. Research Center preprint series \#65, Johns Hopkins University Applied Physics Laboratory, Laurel 1982.

10. C. Beattie and W. M. Greenlee, Convergence theorems for intermediate problems, Proc. Roy. Soc. Edinburgh Sect. A 100 (1985), 107-122. [Corrigenda: Proc. Roy. Soc. Edinburgh Sect. A 104 (1986), 349-350].

11. __ Convergence rates for intermediate problems, Manuscripta Math. 59 (1987), pp. 209227.

12. G. Birkhoff and G. Fix, Accurate eigenvalue computations for elliptic problems, Proc. Numerical Solution of Field Problems in Continuum Physics, Sympos. on Appl. Math., Vol. 2, Amer. Math. Soc., 1970, pp. 111-151.

13. R. D. Brown, Variational approximation methods for eigenvalues: convergence theorems, Computational Mathematics (A. Wakulicz, ed.), Banach Center Publications, Vol. 13, Warsaw, 1984, pp. 543-558.

14. Convergence criterion for Aronszajn's method and for the Bazley-Fox method, Proc. Roy. Soc. Edinburgh Sect. A, 108A (1988), 91-108.

15. G. Fix, Orders of convergence of the Rayleigh-Ritz and Weinstein-Bazley methods, Proc. Nat. Acad. Sci. U.S.A. 61 (1968), 1219-1223.

16. D. W. Fox and J. T. Stadter, An eigenvalue estimation method of Weinberger and Weinstein's intermediate problems, SIAM J. Math. Anal. 8 (1977), 491-503.

17. W. M. Greenlee, Rate of convergence in singular perturbations, Ann. Inst. Fourier (Grenoble) 18 (1968), 135-191.

18. _ Approximation of eigenvalues by variational methods, Communications of the Mathematical Institute, Rijksuniversiteit Utrecht, Vol. 10, Utrecht, 1979.

19. _ A convergent variational method of eigenvalue approximation, Arch. Rational Mech. Anal. 81 (1983), 279-287.

20. P. Grisvard, Charactérisation de quelques espaces d'interpolation, Arch. Rational Mech. Anal. 25 (1967), 40-63.

21. L. Hörmander, Linear partial differential operators, Springer, Berlin-Heidelberg-New York, 1969. 
22. T. Kato, Quadratic forms in Hilbert spaces and asymptotic perturbation series, University of California Press, Berkeley, 1955.

23. __ Perturbation theory for linear operators, Springer, Berlin-Heidelberg-New York, 1966.

24. C. B. Moler and G. W. Stewart, An algorithm for generalized matrix eigenvalue problems, SIAM J. Numer. Anal., 10 (1973), 241-256.

25. L. T. Poznyak, Estimation of the rate of convergence of a variant of the method of intermediate problems, Zh. Vychisl. Mat. i Mat. Fiz. 8 (1968), 1117-1126; English transl., USSR Comput. Math. and Math. Phys. 8 (1968), 246-260.

26. _ The convergence of the Bazley-Fox process, and an estimate of the rate of this convergence, Zh. Vychisl. Mat. i Mat. Fiz. 9 (1968), 860-872; English transl., USSR Comput. Math. and Math. Phys. 9 (1969), 167-184.

27. M. Reed and B. Simon, Methods of modern mathematical physics, Vol. 1, revised and enlarged edition. Academic Press, New York, 1980.

28. _ _ Methods of modern mathematical physics, Vol. 2, Academic Press, New York, 1975.

29. H. F. Weinberger, Error estimation in the Weinstein method for eigenvalues, Proc. Amer. Math. Soc. 3 (1952), 643-646.

30. __ A theory of lower bounds for eigenvalues, Tech. Note BN-103, Inst. for Fluid Dyn. and Appl. Math., Univ. of Maryland, College Park, 1959.

31. _ _ Error bounds in the Rayleigh-Ritz approximation of eigenvectors, J. Res. Nat. Bur. Standards 64B (1960), 217-225.

32. __ Variational methods for eigenvalue approximation, SIAM, Philadelphia, PA, 1974.

33. A. Weinstein and W. Stenger, Methods of intermediate problems for eigenvalues, Academic, Press, New York, 1972.

Department of Mathematics, Virginia Polytechnic Institute and State University, BLACKSBURG, VIRGINIA 24061

E-mail address: beattie@mthunx.math.vt.edu

Department of Mathematics, University of Arizona, Tucson, Arizona 85721 